\title{
Response to the comment on: "Is nuchal cord justified as a cause of obstetrician anxiety?"
}

\author{
Yum Narang • Neelam Bala Vaid · Sandhya Jain • \\ Amita Suneja • Kiran Guleria • M. M. A. Faridi • \\ Bindiya Gupta
}

Published online: 17 October 2014

(C) Springer-Verlag Berlin Heidelberg 2014

To the Editor,

We thank Prof. Gursoy et al. [1] for going through our study [2] in depth and we welcome their comments.

However, here we could like to correct them regarding comment, "pathological range $\mathrm{pH}$ and lactate in non nuchal group in our study". In our study, Nuchal cord group had slightly lower $\mathrm{pH}$ as compared to non-nuchal cord group; however, neonate in neither of the group had pathological range $\mathrm{pH}$ suggesting that presence of $\mathrm{NC}$ interrupt umbilical blood flow to some extent causing some biochemical derangement, however, most with adequately functioning placenta are able to compensate quickly, this may lead to slight fall in $\mathrm{pH}$ but rarely pathological range acidosis in those who are able to sustain vaginal delivery. However, among NC group who were not able to compensate for the reduced blood flow must have had fetal distress and undergone secondary cesarean section. Since we have not included cases of cesarean section in our study, at moment we are not able to comment on this aspect.

This reply refers to the article available at doi:10.1007/s00404-0143516-x.

Y. Narang · N. B. Vaid · S. Jain $(\varangle) \cdot$ A. Suneja $\cdot$ K. Guleria

B. Gupta

Department of Obstetrics and Gynaecology, University College

of Medical Sciences and GTB Hospital, Dilshad Garden,

New Delhi 110095, India

e-mail: sumeet_singla@rediffmail.com;

drsandy2010@rediffmail.com

M. M. A. Faridi

Department of Pediatrics, University College of Medical

Sciences and GTB Hospital, Dilshad Garden, New Delhi, India
Regarding excluding nuchal cord cases delivered by cesarean section. At the start of our study, we purely wanted to evaluate the effect of $\mathrm{NC}$ on outcome parameters of vaginal delivery. Second to avoid bias, as the most common indication for emergency caesarean sections are fetal distress which could be due to meconium staining, non-progress of labour, uterine inertia, second stage arrest, cephalopelvic disproportion, if associated with presence of $\mathrm{NC}$, then NC could be labelled as cause of fetal distress and as indication of caesarean section. NC could be the reason for secondary cesarean section in some cases. Since we only followed vaginal delivery we do not have data on cesarean section due to NC. Further research is underway in our department, after which we can comment on this aspect in a better way.

\section{References}

1. Gursoy AY, Caglar GS (2014) Comment on: Is nuchal cord justified as a cause of obstetrician anxiety? Arch Gynecol Obstet. doi:10.1007/s00404-014-3516-X

2. Narang Y, Vaid NB, Jain S, Suneja A, Guleria K, Faridi MM, Gupta B (2014) Is nuchal cord justified as a cause of obstetrician anxiety? Arch Gynecol Obstet 289(4):795-801. doi:10.1007/ s00404-013-3072-9 